\title{
Molecular mechanics and molecular dynamics analysis of Drexler-Merkle gears and neon pump*
}

\author{
T Cagin $\dagger \S$, A Jaramillo-Botero $\ddagger \|$, G Gao $\dagger \uparrow$ and W A Goddard III $\dagger^{+}$ \\ $\dagger$ California Institute of Technology, MSC, BI, Pasadena, CA, USA \\ ¥ Pontificia Universidad Javeriana, Cali, Colombia
}

Received 6 January 1998

\begin{abstract}
Over the past two years at the Materials and Process Simulation Center, we have been developing simulation approaches for studying the molecular nanomachine designs pioneered by Drexler and Merkle. These nanomachine designs, such as planetary gears and neon pump, are described with atomistic details and involve up to 10000 atoms.

With the Dreiding and universal force fields, we have optimized the structures of the two planetary gear designs and the neon pump. At the Fourth Foresight conference, we reported rotational impulse dynamics studies of the first and second generation designs of planetary gears undergoing very high-frequency rotational motions. We will explore stability of these designs in the lower frequency regimes which require long time simulations. We will report the molecular mechanics and molecular dynamics simulations performed on these model systems. We explore the following modes in these studies: (1) impulse mode; (2) constant angular velocity-perpetual rotation; (3) constant torque-acceleration from rest.
\end{abstract}

$\mathrm{M}$ This article features multimedia enhancements available from the abstract page in the online journal; see http://www.iop.org.

\section{Introduction}

In order to lay a foundation for creating a technology for manufacturing at the nanoscale level, Eric Drexler, Ralph Merkle and collaborators [1-3] have been analyzing designs for mechanical systems at the nanoscale $(1-100 \mathrm{~nm})$ that could in principle be manufactured. We discuss herein some of the design optimization and dynamics simulations, performed on these devices along with their corresponding results and analysis.

\subsection{Nanoscale machines}

At the nanoscale one can no longer think of the material as a continuum whose properties change continuously as it is cut and shaped. Rather one has to consider that it is formed from discrete atoms. Thus at the nanoscale one has a supermolecule rather than a finely divided solid. This requires one to analyze how the elements of normal macromaterials design change as the scale is reduced from

\footnotetext{
$\S$ E-mail address: tahir@wag.caltech.edu

|| Visiting Associate, MSC. E-mail address: ajaramil@wag.caltech.edu

I E-mail address: gao@wag.caltech.edu

+ E-mail address: wag@wag.caltech.edu

* This paper is available on the web at

http://www.foresight.org/Conferences/MNT05/Papers
}

millimeter to micron to nanometers. The differences are dramatic. For a macrosystem a long alkane molecule or a flake of $\mathrm{MoS}_{2}$ might serve as a lubricant, but for a system built on the scale of nm, such molecules may act as dirt that would clog and disable the nanosystem. Similarly the scaling of vibrations, electrical forces, thermal expansion, magnetic interactions and surface tension with size can lead to phenomena very different at the scale of atoms than at the macroscale. Indeed in Nanosystems [2], Drexler considered many aspects of how the scaling of familiar macroscopic concepts changes as one goes to the nanoscale.

In order to make progress here it is necessary to consider specific nanoscale supermolecular systems which could in principle be manufactured. Without worrying about the practical aspects of synthesizing the system, can one make supermolecular assemblies that would function as useful nanoscale machines? Can one design synthetic procedures that could in principle manufacture these machines? Again it is not essential to have a practical costeffective design. It is sufficient to demonstrate the existence theorem, that sequences of steps involving real atoms with the properties of real molecules can indeed lead to processes that would manufacture nanoscale machines with useful functionality. If the aim is achievable, then we can focus on ways to achieve the design in a practical fashion. 


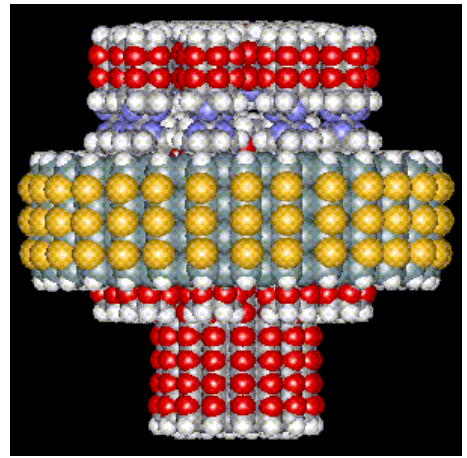

(a)

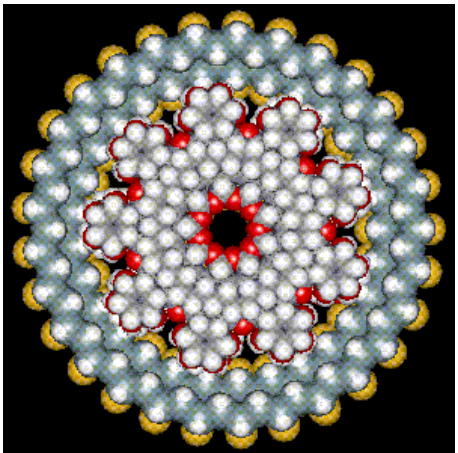

(b)

Figure 1. First generation planetary gear (lateral and top views). Number of atoms: 3557, molecular weight: 51009.8439813 , molecular volume: 33458.272.

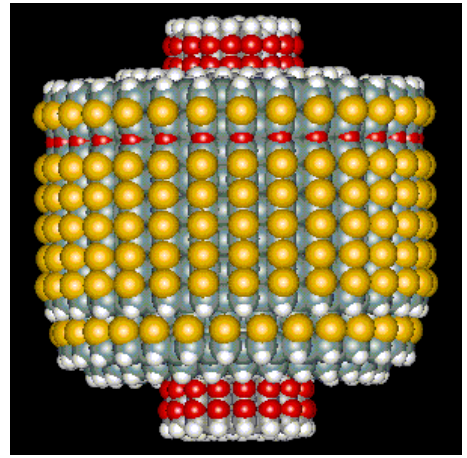

(a)

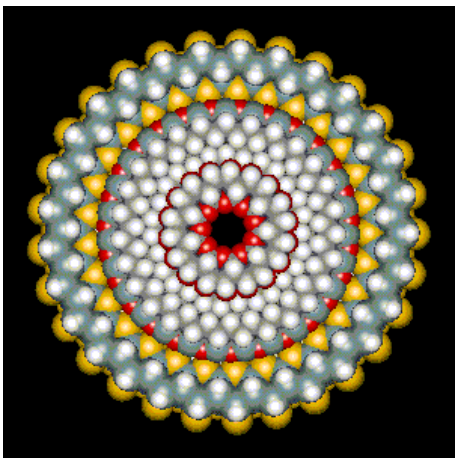

(b)

Figure 2. Second generation planetary gear (lateral and top views). Number of atoms: 4235, exact molecular weight: 72491.9465915 , molecular volume: 47585.964.

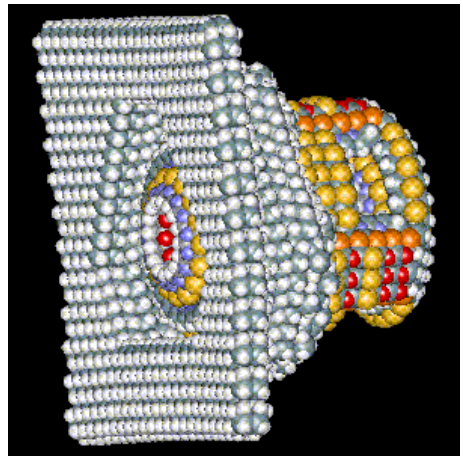

(a)

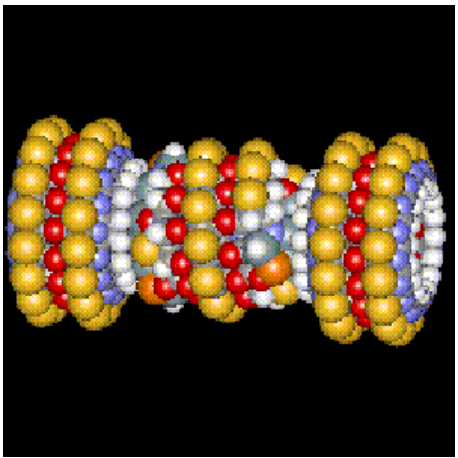

(b)

Figure 3. Neon pump design (a) Complete neon pump. (b) Helical rotor. Number of atoms: 6165 , molecular weight: 88 190.812 6747, molecular volume: 63984.232.

Our ability to model molecular machines (systems and devices)—of specific kinds, designed in part for ease of modeling - has far outrun our ability to make them. Design calculations and computational experiments enable the theoretical study of these devices, independent of the technologies needed to implement them. [2]

\section{Planetary gear}

Along these lines Merkle and Drexler [3] have designed a nanoscale planetary gear with eight moving parts (see figure 1) using molecular mechanics force fields such as DREIDING [4] to ensure that the bond 
Neon Pump: rotation (360 degrees)

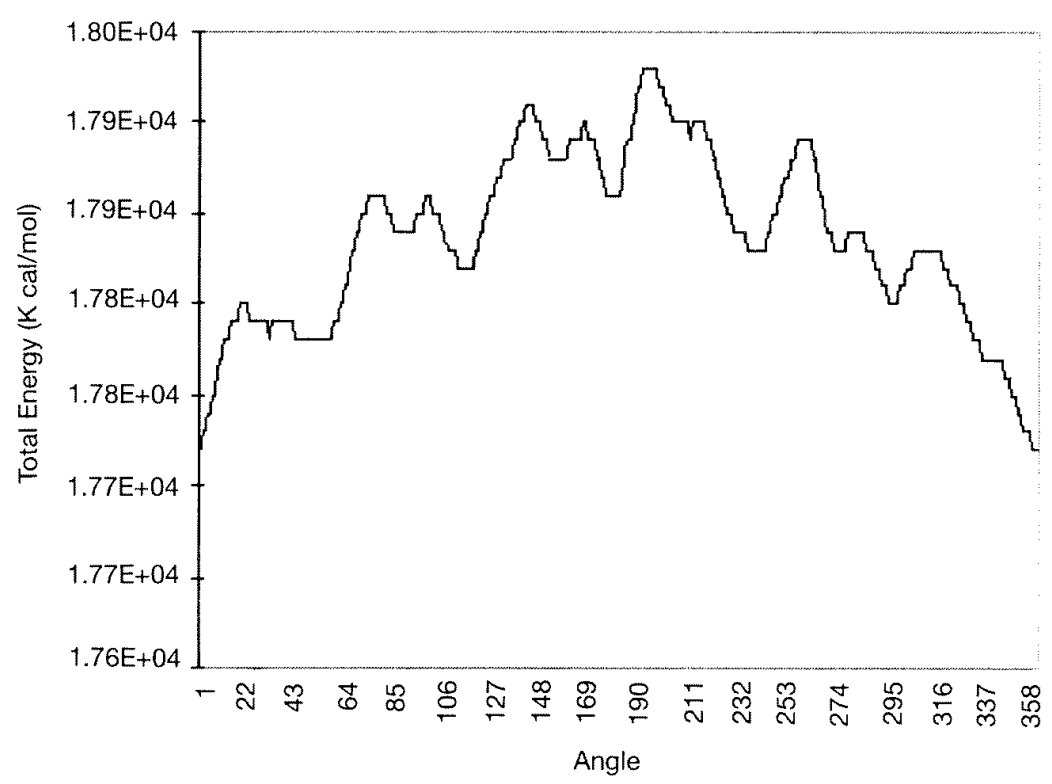

Figure 4. Energy curve during $360^{\circ}$ rotation shows helix interactions and slight rotor deformation after minimization of structure (no Ne).

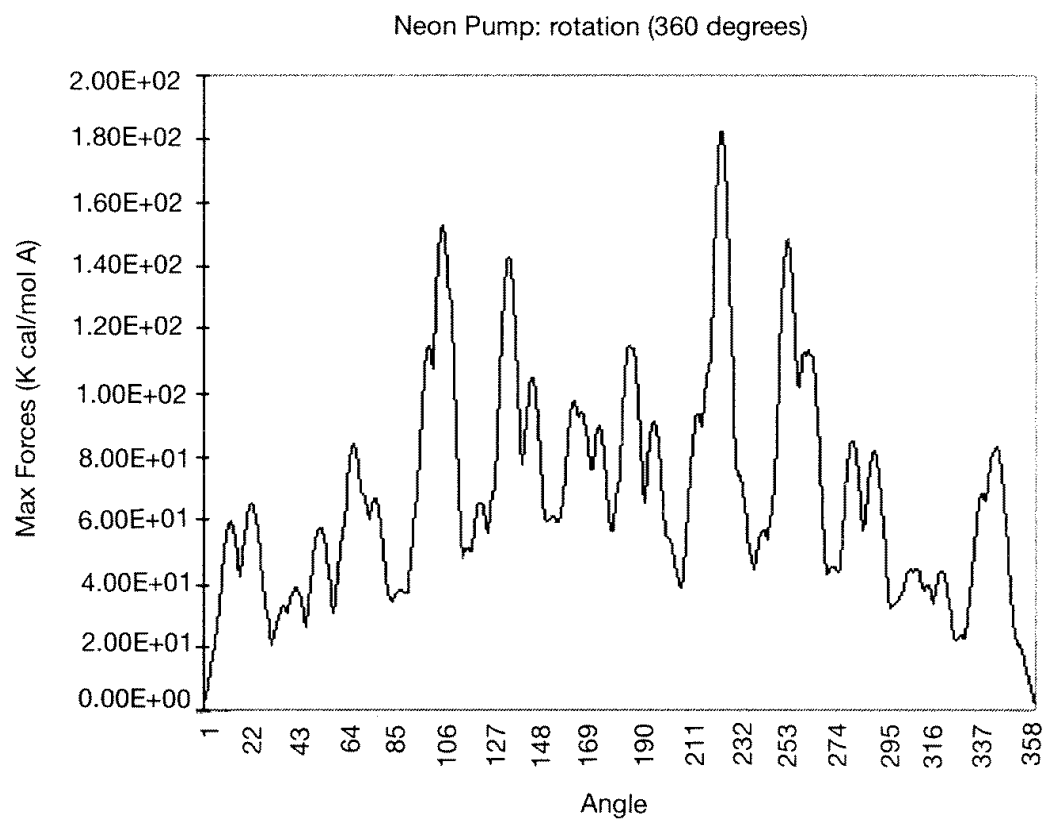

Figure 5. Force levels during the rotation show the magnitude of forces required to set the system in motion through an externally applied force directly on the rotor or by indirect forces induced by high pressure (no $\mathrm{Ne}$ ).

lengths, bond angles, van der Waals distances and strain energies are reasonable. Based on their design we carried out and reported at the 1995 Nanotechnology conferences a molecular dynamics simulation to test the properties of this gear [5]. Using somewhat extreme conditions (rapid accelerations and high-frequency rotations), we found significant instabilities in this design. In response Merkle and Drexler [6] improved their designs (see figure 2) to achieve much more stable motions.

\section{Observed results}

We find that this new design is more stable; however, it also has too much slip. That is, the actual distances between the atoms for the specific set of atoms in this supermolecular system lead to spacings between the gear teeth and the opposing gear faces that are easily stripped. The problem is that a careful balance must be achieved between:

- having the gear and race atoms so close that shortrange repulsive van der Waals interactions cause the gear to freeze, and 


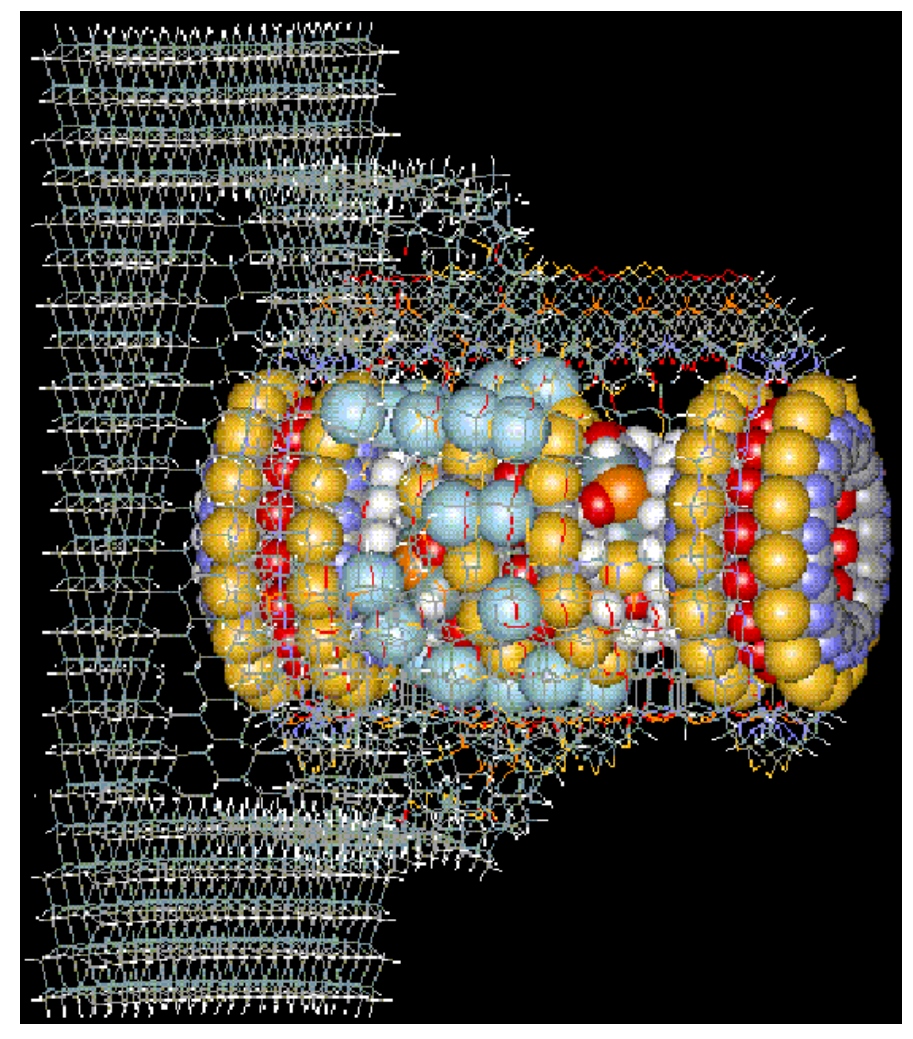

Figure 6. Actual trajectory of neon atoms (blue: 28) during pumping action.

M An animated gif of this figure is available from the article's abstract page in the online journal; see http://www.iop.org. (a) Energy $(\mathrm{K} \mathrm{cal} / \mathrm{mol})$

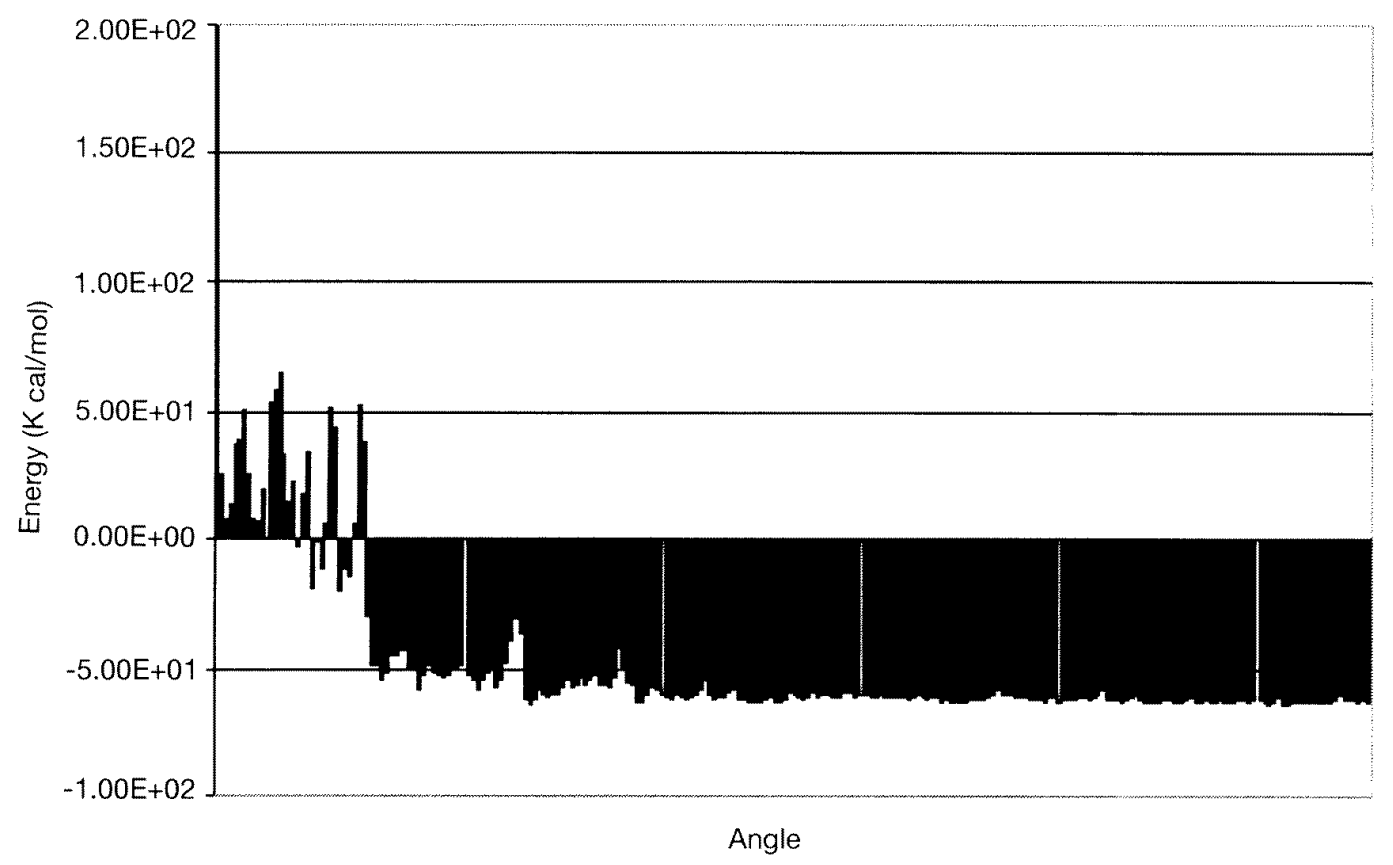

Figure 7. Results of 3.4 revolutions with $28 \mathrm{Ne}$ atoms in the pump chamber: $(a)$ total energy, $(b)$ maximum forces, (c) RMS forces. 
(b)

RMS Force ( $\mathrm{K} \mathrm{cal} / \mathrm{mol} \mathrm{A})$ along 3.4

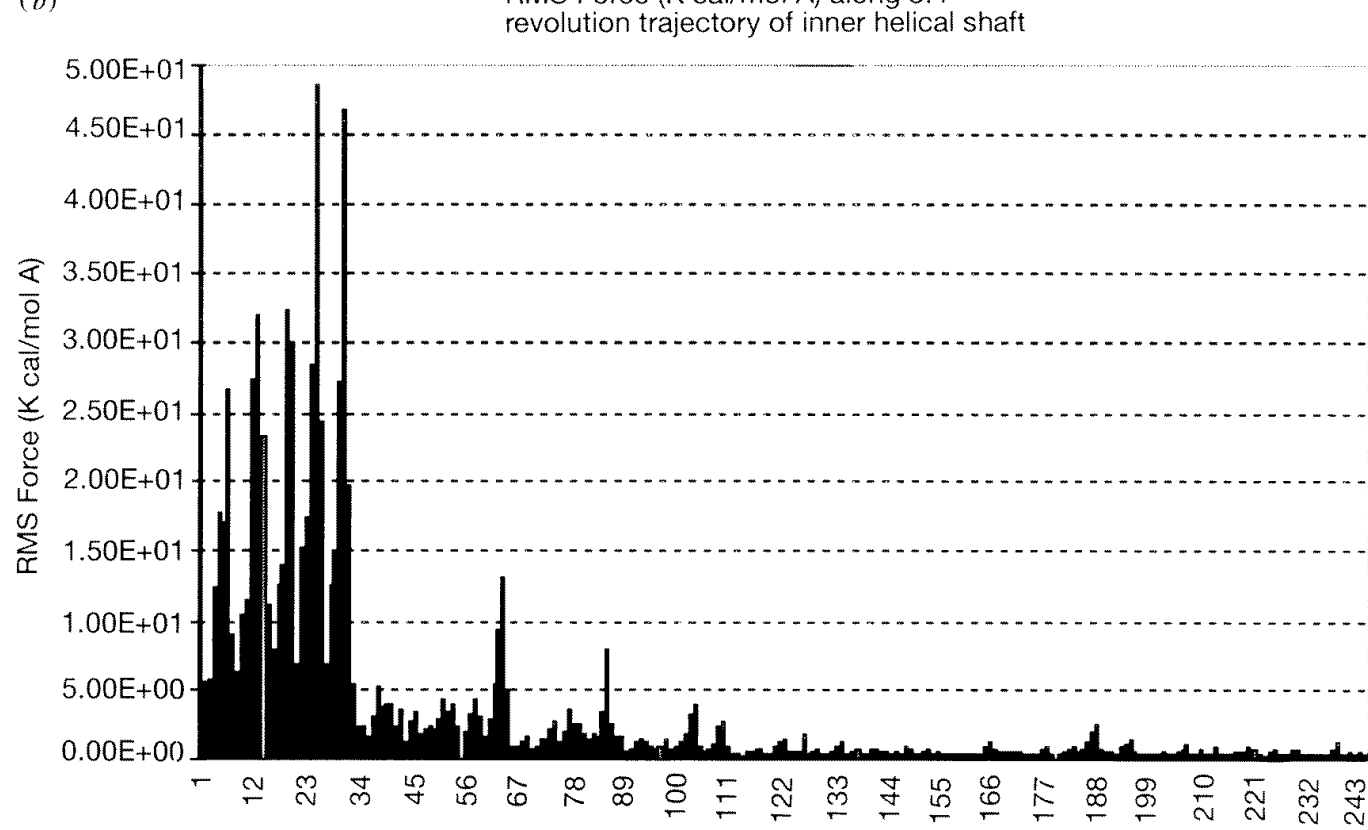

Rotation Step

(c)

Maximum force along same 3.4

revolution trajectory of inner helical shaft

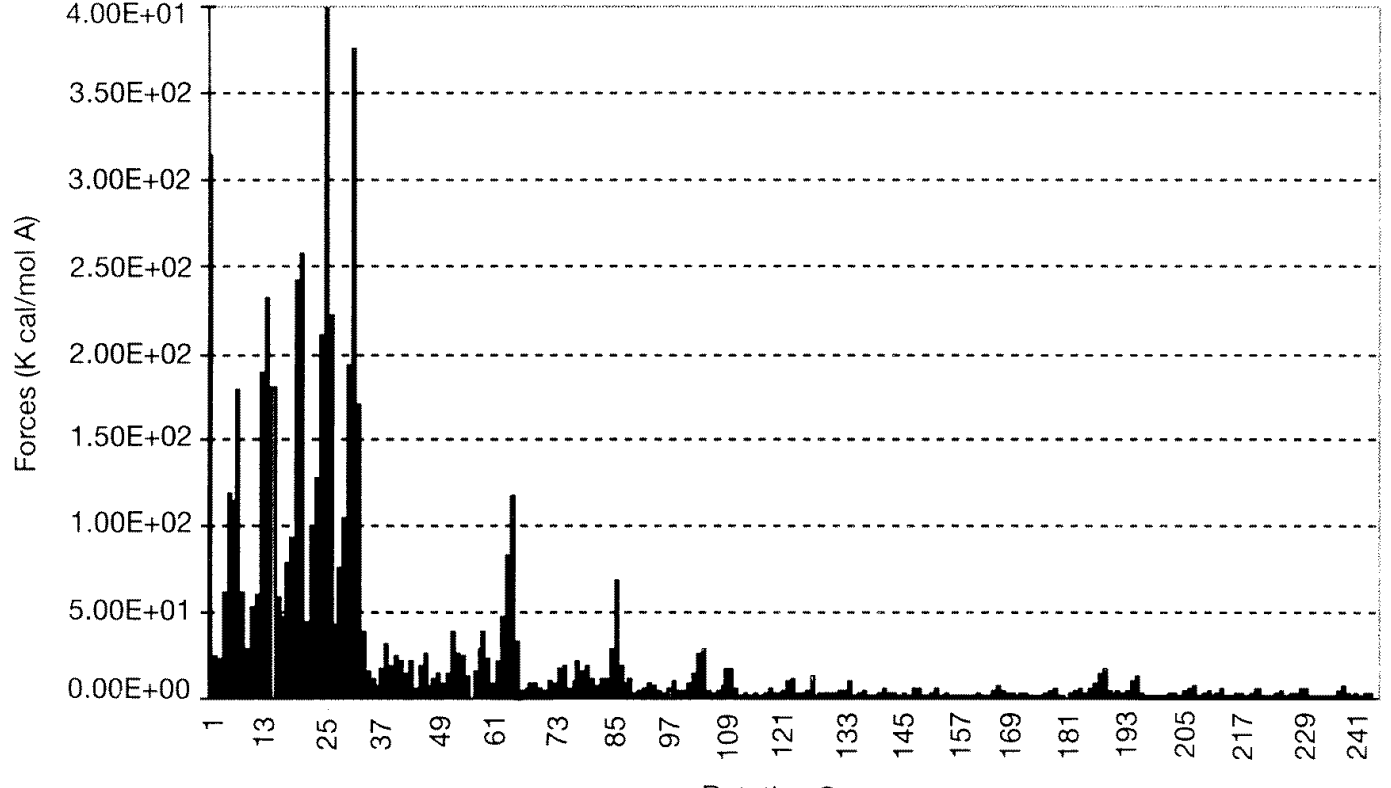

Rotation Step

Figure 7. (Continued)

- having them too far apart that the gear tooth can slip past the gear tooth of the race.

It is possible to balance these in the static system, but dynamical motions lead to fluctuations (vibrations) that allow slippage. This is not a calamity and does not mean that nanotechnology will not work. It means that we have a lot of hard work to do in designing prototype systems and testing them.

The problem here is the discreteness of the atoms. A macroscopic system is treated as a continuum. To make a gear one can design the shape of the gear teeth and the shape of the opposing teeth of the face to have exactly the same spacing as the gears turn. It is then only necessary to design the casting or injection molding system with appropriate tolerances to achieve good performance. The discreteness of the atoms plays no role when these tolerances are $0.0001 \mathrm{in}=2.54 \times 10^{-3} \mathrm{~mm}$. 


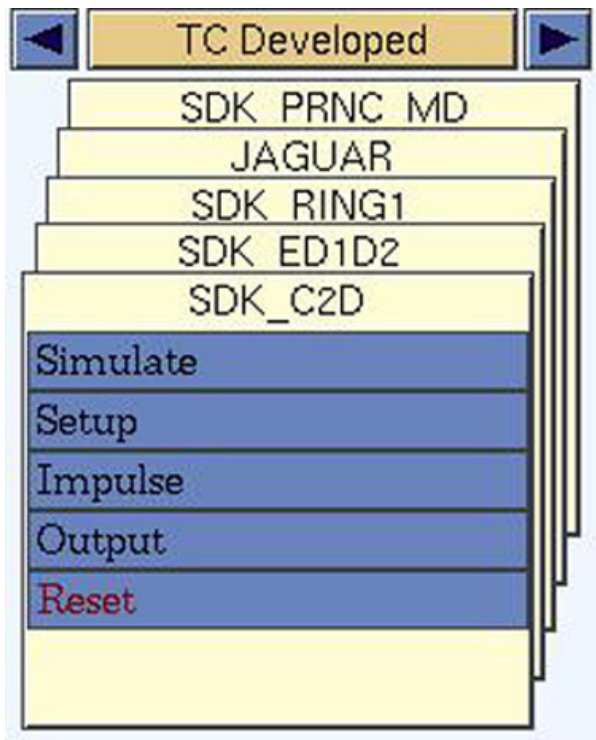

(a)

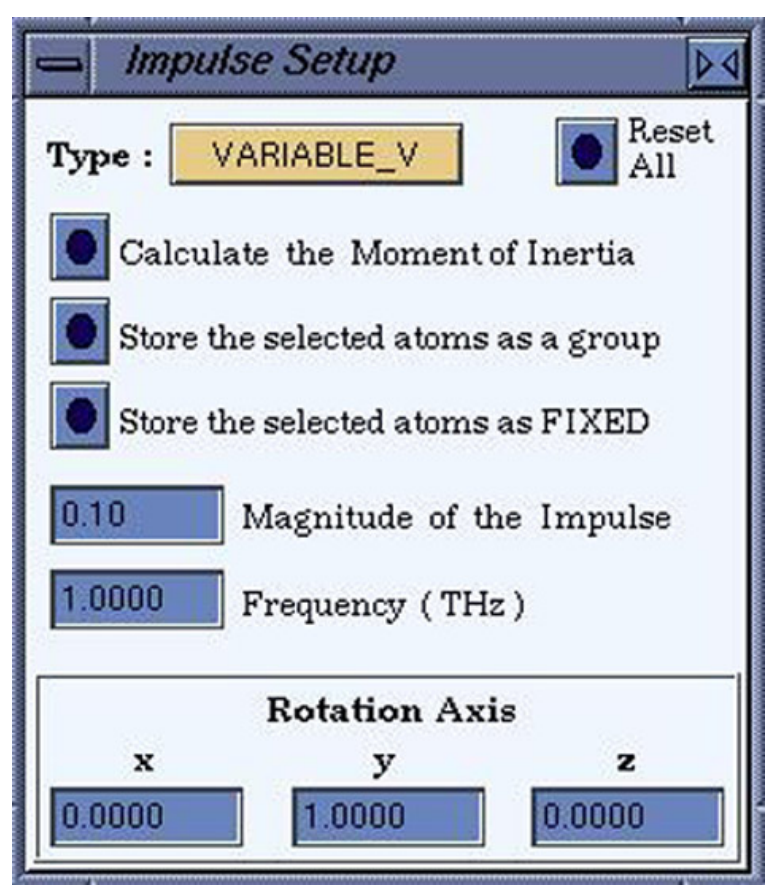

(b)

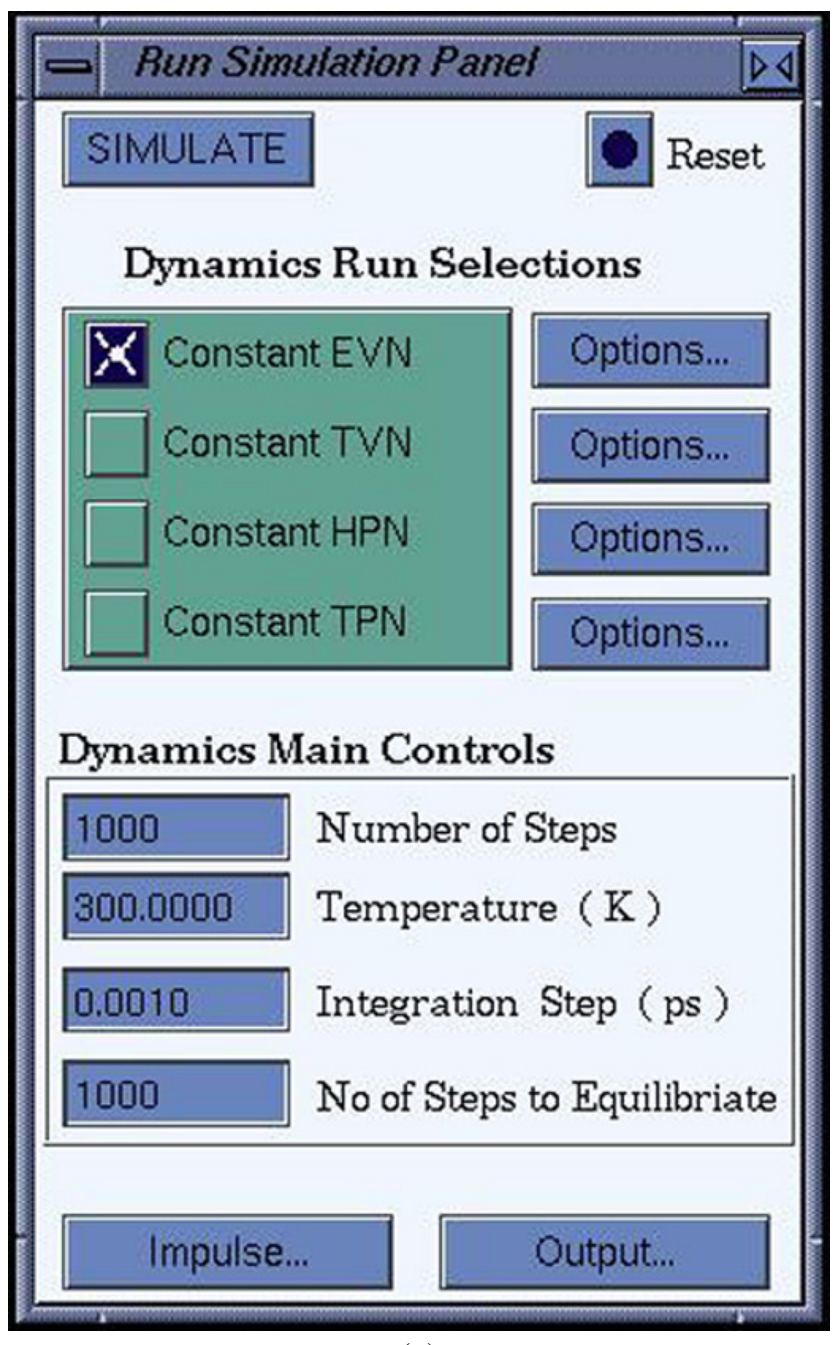

(c)

Figure 8. Cerius $^{2}$ graphical user interface to nanomachine simulator. (a) Impulse set-up, (b) dynamics, (c) run simulation panel. 


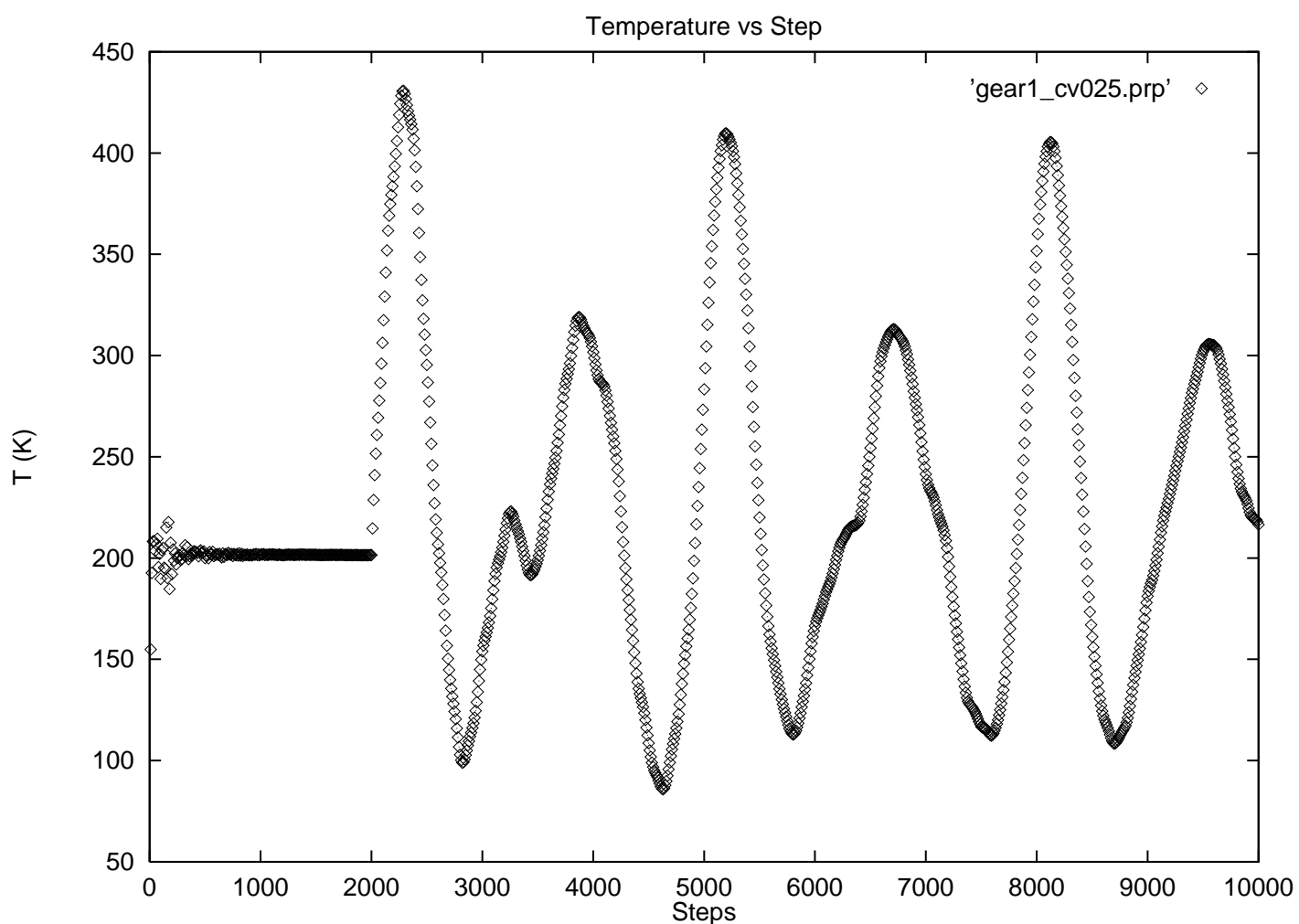

(a)

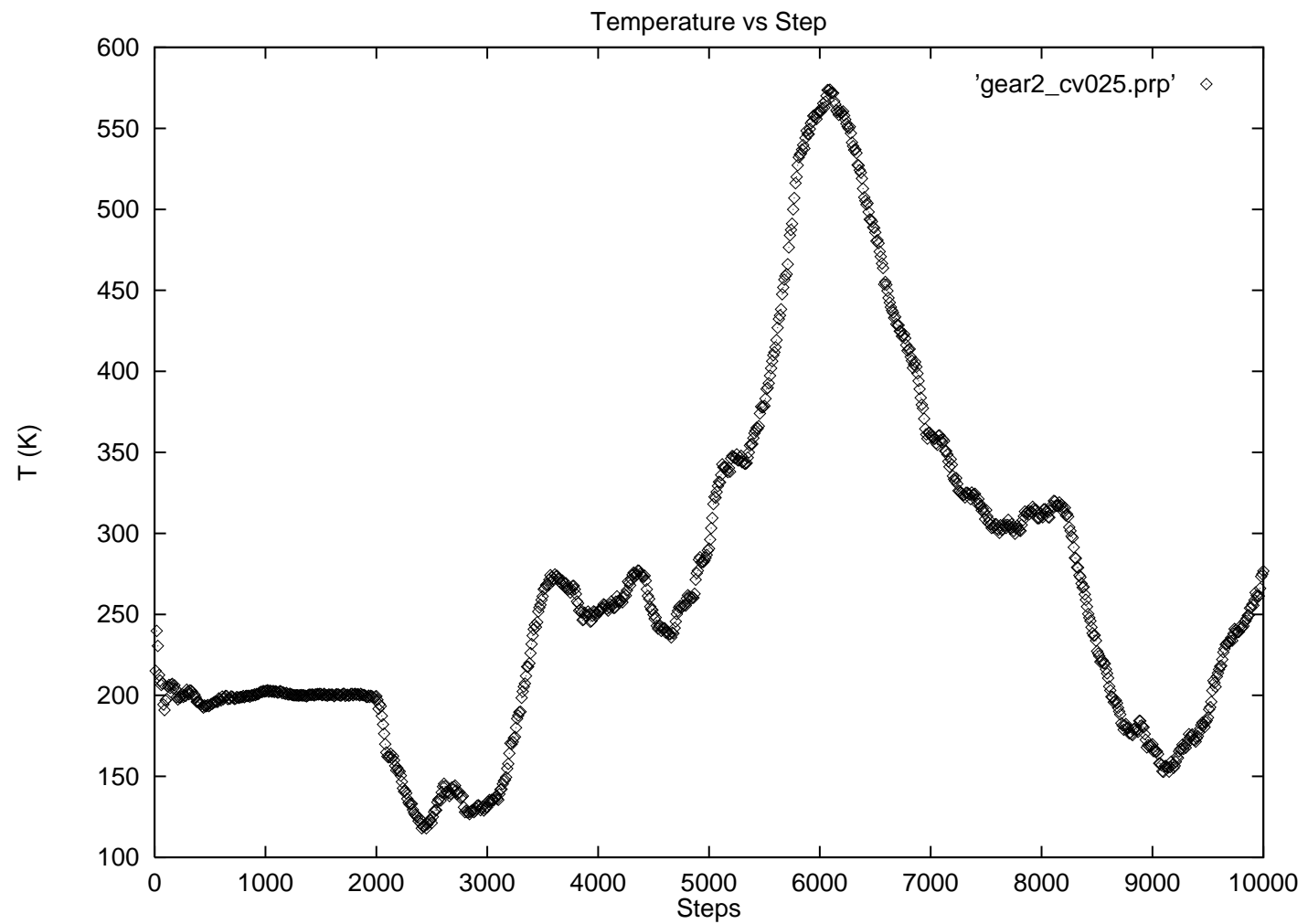

(b)

Figure 9. Kinetic temperature versus different driving conditions. (a) Kinetic temperature of generation 1 planetary gear driven by constant initial angular velocity. For the initial 2000 iterations the kinetic energy is maintained at a constant value. (b) Kinetic temperature of generation 2 planetary gear driven by constant angular velocity. For the initial 2000 iterations the kinetic energy is maintained at a constant value. (c) Kinetic temperature of generation 2 planetary gear driven by a sine wave angular velocity. For the initial 2000 iterations the kinetic energy is maintained at a constant value. (d) Cold start with a sinusoidal driving torque applied. 


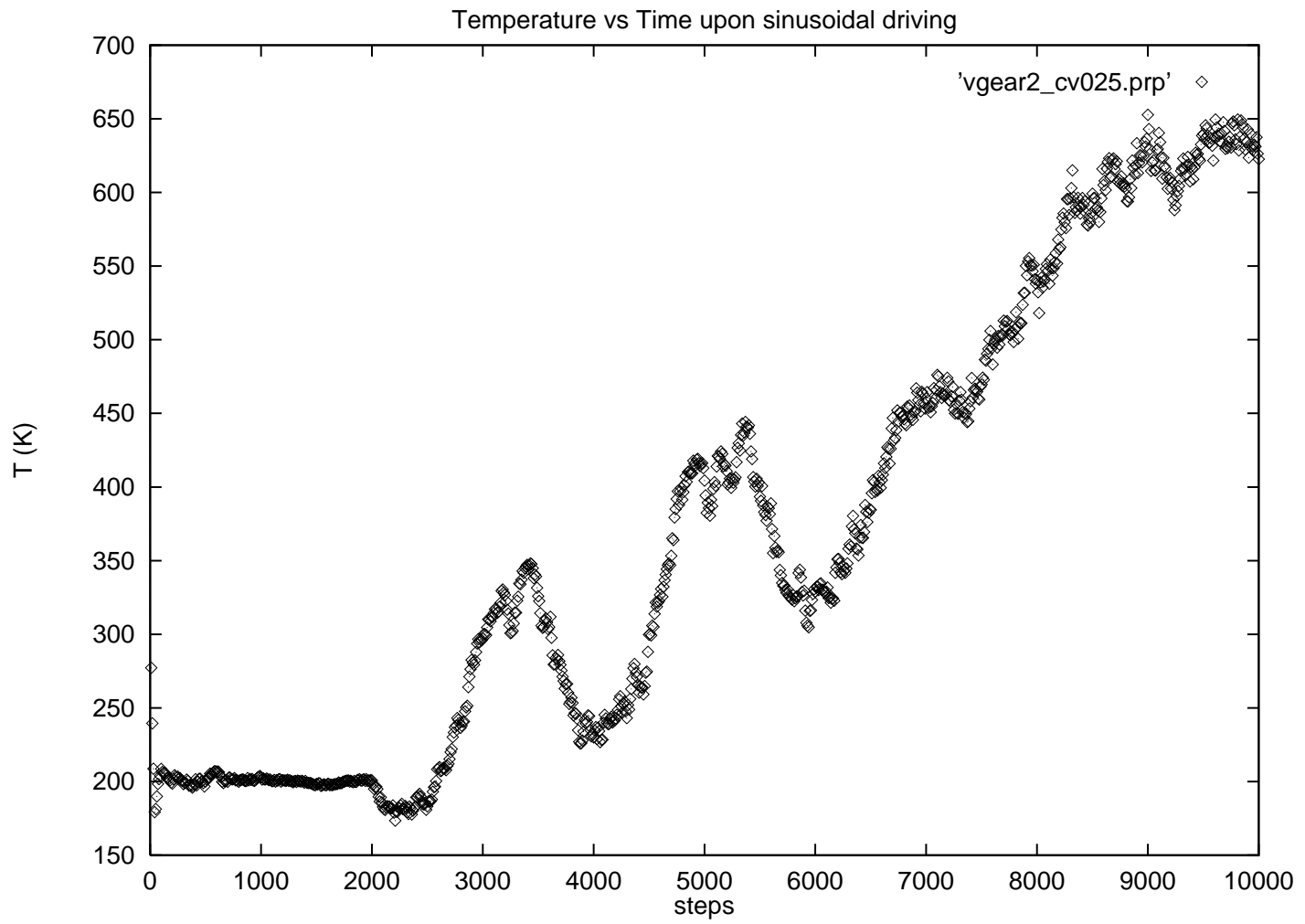

(c)

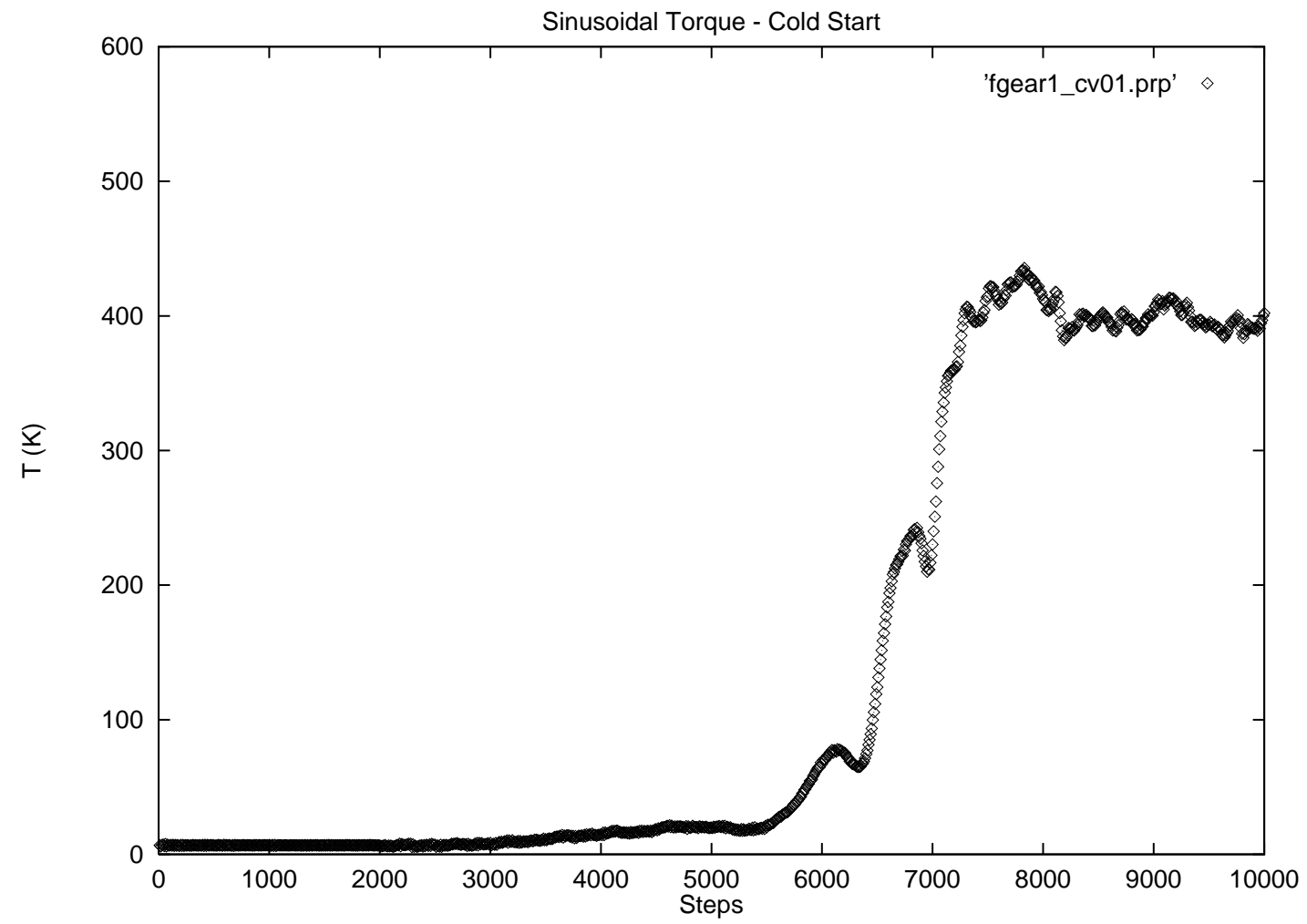

(d)

Figure 9. (Continued) 


\section{Neon pump}

More recently Drexler and Merkle [3] have designed a nanomachine (figure 3) which in one mode could serve as a pump for $\mathrm{Ne}$ and in another could serve to convert $\mathrm{Ne}$ pressure to rotary power (figure 6). We investigate this system using accurate force fields and molecular dynamics simulations to test and refine the concepts.

\subsection{Static analysis}

The initial tests performed on the neon pump involve a $0 \mathrm{~K}$ simulation, using universal force fields (UFF) [7], and partial charges obtained from the Gasteiger method, to establish the effect of rotor-casing, rotor-casing-Ne interactions. The following test where performed.

- Rotor static rotations with single energy calculations at each step, to determine the magnitude of forces required for inducing motion on the rotor and to determine possible empty system 'frictions' along the trajectory (see figures 4 and 5).

- Translations along the axis of rotation were performed to evaluate energy curves during rotation and push-pull action induced by pumping action. This analysis serves as well to determine kinematic-dynamic boundaries of the system, i.e. maximum angular velocities, acceleration, etc.

- Neon atoms are introduced into the chamber of the pump and step by step $1^{\circ}$ rotations of the helix followed by energy minimizatons at each step (while fixing atomic positions in the rotor and casing to avoid all atomic computation of the equations of motion, i.e. the main interest is in $\mathrm{Ne}$ trajectories).

In a sense, we can consider these simulations as being two rigid body (rotor-casing) and multiple body (rotorcasing-Ne atoms) (figure 7).

Simulation shows a total of 245 rotation steps $\left(5^{\circ}\right.$ each), for a total of 3.4 revolutions. Results clearly show higher energy due to $\mathrm{Ne}-\mathrm{Ne} / \mathrm{Ne}-$ rotor/ $\mathrm{Ne}-$ casing interactions during their motion through the helix. The number of $\mathrm{Ne}$ atoms inside the helix chamber reduces as the rotations step axis increases. Energy becomes negative since only $\mathrm{Ne}$ atoms are considered.

The ground rules here are that a realistic force field (FF) be used and that all pieces be treated at the atomic level (but some might be semi-rigid). This will use the FF developed for nanosynthesis. Also we wish to consider the effect of energy release in the chemical steps on the thermal fluctuations in these systems (which may cause displacements and vibrations).

\section{Molecular dynamics simulations using rotational impulse dynamics}

The molecular dynamics simulation is initiated by a rotational impulse to the input unit. In the course of simulation this angular momentum is slowly transferred to the output unit.

Over the $12.0 \mathrm{ps}$ simulation, the input unit completes one full cycle and the output unit completes almost a half cycle and the rail completes a quarter cycle. Over the run the initial angular kinetic energy partially dissipates through conversion into thermal energy via atomic collisions.

We imagine that the eventual design would have the functionality of a planetary gear but might have an appearance completely different from the macroscopic systems. Thus because a gear tooth in the $x y$ plane cannot be atomically smooth in the $z$-direction, we may develop a Vee design so that the Vee shape of the gear tooth in the $z$-direction nestles within a Vee notch in the race to retain stability in the $z$-direction as the teeth contact in the $x y$ plane. This design would make no sense for a macroscopic gear system since the gear could never be placed inside the race. However, for a molecular system one could imagine that the gear is constructed and that the race is constructed all except for a last joining unit. The parts could be assembled and then the final connections on the face made to complete the design.

The preliminary tests performed on the neon pump demonstrate an appropriate functional behavior. The structural deformations of the rotor can cause instabilities at low and high rotational frequencies. The forced translations show that at very low perpendicular forces due to pump action the total energy rises significantly and again the structure deforms.

\section{Control of power input for nanogears and pumps}

For all three of the devices we study, the driver unit is a rotary component. In order to mimic operational conditions, we designed various power input profiles to drive the devices. Under such conditions these devices are not represented by thermodynamic equilibrium, but rather they are driven systems represented as in steady state thermodynamic systems.

The modes of input power profiles we used are as follows.

(1) Impulse mode. A rotational angular velocity is applied to the input unit at $t=0$.

(2) Constant angular velocity. The input unit is maintained at a constant rate of rotation about the chosen axis. Unless a heat sink is supplied (Nose-Hoover thermostat or Gaussian isokinetic thermostat, or Langevin bath) the model system heats up, and is driven away from the steady thermodynamic state.

(3) Time-dependent angular velocity. The input unit is driven by a time-dependent angular velocity. The functional forms for time dependence should be bounded functions, especially periodic functions, sine, saw tooth, etc. This mode of operation must also be maintained at a steady state by a heat bath.

(4) Constant torque-acceleration from rest. This is especially important to simulate the early stages of the operation. Where a constant angular acceleration rate starts the system from rest.

(5) Time-dependent angular acceleration mode. This mode is similar to that of the time-dependent angular velocity profile imposed on the input unit. Especially, periodic functions for input torque cause the system to remain within stability limits. 


\section{T Cagin et al}

We have implemented these driving modes into the Cerius $^{2}$ program environment [8] using the software developers kit [9] version 3.0. The module utilizes graphics, communication, user interface and data model subsystems of Cerius ${ }^{2}$. In this implementation, the user may choose any one of the energy and force evaluators. It can either be the Cerius ${ }^{2}$ open force field module or an independent energy and force evaluator (user developed). In our computations we used the Cerius ${ }^{2}$ OFF module. This development is an attempt to supply a nanomachine specific simulation module [10] which could be used by various nanotechnologists, see figure 8 .

\subsection{Dynamics simulation of gears and neon pump}

The simulations are performed at various input conditions: constant angular velocity and constant angular acceleration for both gear designs and neon pump. We have also utilized sinusoidal driving conditions:

$$
W(t)=W_{0} \sin (\omega t)
$$

$W$ can either be angular velocity or angular acceleration, $\omega$ is the frequency of the power source. We especially investigated the temperature profile as a function of time. To see if the system is driven too far from equilibrium we turned off the temperature control. (Under these conditions a power source exists, but no sink mechanism is turned on.) The time variation of the kinetic temperature of various systems and driving conditions are given in figure 9.

\section{Concluding remarks}

Of course, we know that nanoscale machines are possible. Nature has devised methods of synthesizing quite remarkable nanoscale machines that function reliably, are synthesized cheaply and reliably (often self-correcting) from readily available materials and energy sources, and are environmentally benign (biodegradable). Indeed modern science is finding ways to manipulate these systems to manufacture modified molecules and systems with predesigned properties and functionalities. However, the complex chemistry and biology involved in such systems limits the applications and flexibility to modify these systems for dramatically different applications. Thus it is important to determine the feasibility of designing and making nanoscale machines with new functionality and characteristics.

\section{References}

[1] Drexler K E 1986 Engines of Creation

[2] Drexler K E 1992 Nanosystems: Molecular Machinery, Manufacturing, and Computation

[3] Drexler K E and Merkle R First Generation Planetary Gear

[4] Mayo S L, Olafson B D and Goddard W A III 1990 DREIDING: A generic force field for molecular simulations J. Phys. Chem. 948897

[5] Gao G, Cagin T and Goddard W A III 1995 Nanotechnology Conf. Sponsored by the Foresight Institute, (October) (MD Simulation of the Drexler-Merkle Nanoscale Planetary Gear) (Palo Alto, CA)

[6] Drexler K E and Merkle R 1995 Second generation planetary gear 1995 Nanotechnology Conf. Sponsored by the Foresight Institute (October) (Palo Alto, CA)

[7] Rappe A K, Casewit C J, Colwell K S, Goddard W A III and Skiff W M 1992 J. Am. Chem. Soc 114 10024-35

[8] Cerius ${ }^{2}$ program Molecular Simulations Incorporated (San Diego, CA)

[9] 1997 Software developers kit, version 3.0 Molecular Simulations Incorporated

[10] Cagin T 1997 NanoSim: A Nanomachine Simulation Program. This is a nanosystem simulation program interfaced to Cerius ${ }^{2}$ program environment using software developers kit. 